\title{
Role of Acid-Base Equilibria in the Size, Shape, and Phase Control of Cesium Lead Bromide Nanocrystals
}

\author{
Guilherme Almeida, ${ }^{\dagger, \perp}$ Luca Goldoni, ${ }^{\ddagger}, \S$ Quinten Akkerman, ${ }^{\dagger, \perp}$ Zhiya Dang, ${ }^{\dagger}$ Ali Hossain Khan,
} Sergio Marras, $"$ Iwan Moreels, ${ }^{\dagger, \#[0}$ and Liberato Manna ${ }^{*}{ }^{\dagger}$ (i)

${ }^{\dagger}$ Department of Nanochemistry, ${ }^{\dagger}$ D3-PharmaChemistry Line, ${ }^{\S}$ Analytical Chemistry Facility, and "Materials Characterization Facility, Istituto Italiano di Tecnologia, Via Morego 30, 16163 Genova, Italy

${ }^{\perp}$ Dipartimento di Chimica e Chimica Industriale, Università degli Studi di Genova, Via Dodecaneso, 31, 16146 Genova, Italy

\#Department of Chemistry, Ghent University, Krijgslaan 281-S3, 9000 Gent, Belgium

\section{Supporting Information}

ABSTRACT: A binary ligand system composed of aliphatic carboxylic acids and primary amines of various chain lengths is commonly employed in diverse synthesis methods for $\mathrm{CsPbBr}_{3}$ nanocrystals (NCs). In this work, we have carried out a systematic study examining how the concentration of ligands (oleylamine and oleic acid) and the resulting acidity (or basicity) affects the hotinjection synthesis of $\mathrm{CsPbBr}_{3}$ NCs. We devise a general synthesis scheme for cesium lead bromide NCs which allows control over size, size distribution, shape, and phase $\left(\mathrm{CsPbBr}_{3}\right.$ or $\left.\mathrm{Cs}_{4} \mathrm{PbBr}_{6}\right)$ by combining key insights on the acid-base interactions that rule this ligand system. Furthermore, our findings shed light upon the solubility of $\mathrm{PbBr}_{2}$ in this binary ligand system, and plausible mechanisms are suggested in order to understand the ligandmediated phase control and structural stability of $\mathrm{CsPbBr}_{3} \mathrm{NCs}$.

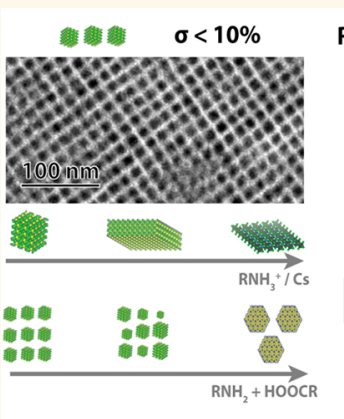
$\mathrm{RNH}_{2}+\mathrm{HOOCR} \stackrel{\mathrm{K}}{\rightleftharpoons} \mathrm{RNH}_{3}^{+}$-OOCR

$\mathrm{CsPBr}_{3}$ $\left[\mathrm{RNH}_{3} \mathrm{P}_{2} \mathrm{PbBr}_{4}\right.$ $\mathrm{Cs}_{4} \mathrm{PbBr}_{6}$

KEYWORDS: halide perovskites, $\mathrm{Cs} P b \mathrm{Br}_{3}$, nanocrystals, acid-base, colloidal synthesis

$\mathrm{L}$ ead halide perovskites (LHPs), with the formula $\mathrm{APbX}_{3}$ ( $\mathrm{A}=\mathrm{CH}_{3} \mathrm{NH}_{3}$, Cs etc; $\left.\mathrm{X}=\mathrm{Cl}, \mathrm{Br}, \mathrm{I}\right)$, have been extensively researched over the past decade as active absorber materials for solar cells, with efficiencies now exceeding $22.1 \% .^{1-3}$ Following the striking performances of thin-film LHPs, $\mathrm{CsPbX}_{3}$ nanocrystals (NCs) were synthesized by Protesescu et al. ${ }^{4}$ These $\mathrm{CsPbX}_{3}$ NCs possess bright and easily tunable luminescence over the entire visible range, which makes them excellent candidates for photonic sources. ${ }^{4-6}$ With respect to the traditional semiconductors that are typically used in these applications, LHPs offer potentially lower manufacturing costs by relying on solution-based processes. ${ }^{7}$ However, still little is known about the underlying mechanisms that rule their formation, even though they are the key to improving quality standards and to the development of commercial formulations. $^{8-12}$

Generally, LHP thin films, powders, and NCs are fabricated by precipitation reactions in aprotic solvents. In particular, the synthesis of NCs is conducted in a nonpolar solvent and often in the presence of a binary ligand system composed of aliphatic carboxylic acids and primary amines of various chain lengths. ${ }^{4,13}$ In the common hot-injection synthesis of cube-shaped $\mathrm{CsPBr}_{3}$
$\mathrm{NCs},{ }^{4}$ Cs-oleate is injected into a hot solution $\left(190{ }^{\circ} \mathrm{C}\right)$ of $\mathrm{PbBr}_{2}$, oleic acid (OA), oleylamine (OlAm), and 1-octadecene (ODE). Although this approach leads to strongly luminescent NCs, reports have also indicated a fast defocusing of the size distribution within a few seconds from the injection, ${ }^{14}$ making it difficult to obtain samples with standard deviations below $15 \%$, which are highly desirable for both device fabrication and fundamental studies. Moreover, size control was limited (8-12 $\mathrm{nm}$ ) by the narrow workable temperature range, which was bound by the precipitation of $\mathrm{PbBr}_{2}$ at ca. $195{ }^{\circ} \mathrm{C}$ and by the coformation of nanoplatelets (NPLs) below $150{ }^{\circ} \mathrm{C}$. $4,8,15$ Furthermore, accurate shape and thickness control of NPLs is yet to be achieved using the hot-injection method. ${ }^{8,15}$ In this regard, a clearer understanding of the factors that regulate the formation of NPLs is needed in order to improve their quality. So far, their formation has not only been correlated to low temperatures ${ }^{15}$ but also to the presence of short alkylamines ${ }^{8}$

Received: November 25, 2017

Accepted: January 30, 2018

Published: January 30, 2018 
and high concentrations of alkylammonium ions, ${ }^{16,17}$ but there is no clear consensus on what the key driving factor is.

In this work, we demonstrate how the acid-base chemistry of the OlAm/OA binary ligand system is central to the size, shape, and phase control of cesium lead bromide NCs synthesized via the hot-injection method. We have found that increasing the concentration of ligands promotes Ostwald ripening but also allows the precipitation temperature of $\mathrm{PbBr}_{2}$ to rise above $195{ }^{\circ} \mathrm{C}$ and to enable the synthesis of NCs in a temperature range (above $195{ }^{\circ} \mathrm{C}$ ) that has hardly been explored to date. Furthermore, we confirmed that mixtures of oleic acid and oleylamine in a nonpolar medium lead to the protonation of oleylamine by oleic acid. Oleylammonium-rich synthetic conditions promoted anisotropic growth through a competition between oleylammonium and $\mathrm{Cs}^{+}$ions. The concentration of oleylammonium $\left(\mathrm{RNH}_{3}{ }^{+}\right)$could be increased not only by raising the concentration of oleic acid but also by lowering the temperature. By combining these key insights, we were able to prepare nanocubes from $4.0 \mathrm{~nm}$ (strongly quantum confined) to $16.4 \mathrm{~nm}$ (a range which is significantly wider than the $8-12 \mathrm{~nm}$ obtained by the standard approach ${ }^{4}$ ), with size distributions in the range of 8 to $15 \%$. Larger sizes were also accessible, at the expense of a broader size distribution. Similarly, we could synthesize NPLs with controlled thicknesses, down to a single monolayer sheet with a $\left(\mathrm{RNH}_{3}\right)_{2} \mathrm{PbBr}_{4}$ composition. The phase of the NCs could be switched from $\mathrm{CsPBr}_{3}$ to the so-called zerodimensional $\mathrm{Cs}_{4} \mathrm{PbBr}_{6}$, when working above a threshold concentration of ligands. This is explained in terms of the high solubility of $\mathrm{PbBr}_{2}$ at high concentration of ligands, which shifts the $4 \mathrm{CsPbBr}_{3} \leftrightarrow \mathrm{Cs}_{4} \mathrm{PbBr}_{6}+3 \mathrm{PbBr}_{2}$ equilibrium to the right. Finally, many of these findings could be successfully extended to the $\mathrm{Cl}$ and I systems.

\section{SIZE, SHAPE, AND PHASE CONTROL OF CESIUM LEAD BROMIDE NCS}

In this section, we will address the synthesis of NCs, specifically the acid-base conditions which enable control over size, shape, and phase. Mechanistic considerations will be discussed in the following sections. The $\mathrm{Cs} \mathrm{PbBr}_{3} \mathrm{NCs}$ reported in this work were all synthesized based on the method reported by Protesescu et al., ${ }^{4}$ and the size and shape control was obtained by only altering the reaction temperature and the concentrations of $\mathrm{OA}, \mathrm{OlAm}$, and $\mathrm{Cs}^{+}$. In short, $72 \mathrm{mg}$ of $\mathrm{PbBr}_{2}(0.20$ mmol, $33 \mathrm{mM}$ ) were dissolved in a $6.0 \mathrm{~mL}$ mixture of various concentrations of ligands (OlAm and OA) and solvent (ODE). After the $\mathrm{PbBr}_{2}$ was dissolved at $100{ }^{\circ} \mathrm{C}$ under vacuum, the mixture was heated under nitrogen to the target reaction temperature, at which point $0.5 \mathrm{~mL}$ of a warm Cs-oleate solution was injected. This resulted in an instantaneous nucleation burst. Thereafter, samples were immediately cooled to room temperature with an ice bath and separated by centrifugation (see the Methods for additional details).

It is important to note that $\mathrm{PbBr}_{2}$ is highly soluble in the binary ligand mixture (OlAm + OA) but not in OA or OlAm alone (as will be further discussed later). Under the conditions that are proposed by Protesescu et al. $([\mathrm{OA}]=[\mathrm{OlAm}]=0.25$ $\mathrm{M}), \mathrm{PbBr}_{2}$ precipitates when it is heated to a threshold temperature of $195^{\circ} \mathrm{C}(\mathrm{XRD}$ pattern shown in Figure $\mathrm{S} 1$ of the Supporting Information, SI). ${ }^{4}$ By increasing the amount of ligands, we found that this precipitation temperature can be increased up to $290^{\circ} \mathrm{C}$, as shown in Figure 1, which allows for the synthesis of $\mathrm{CsPbBr}_{3} \mathrm{NCs}$ even at high temperatures.

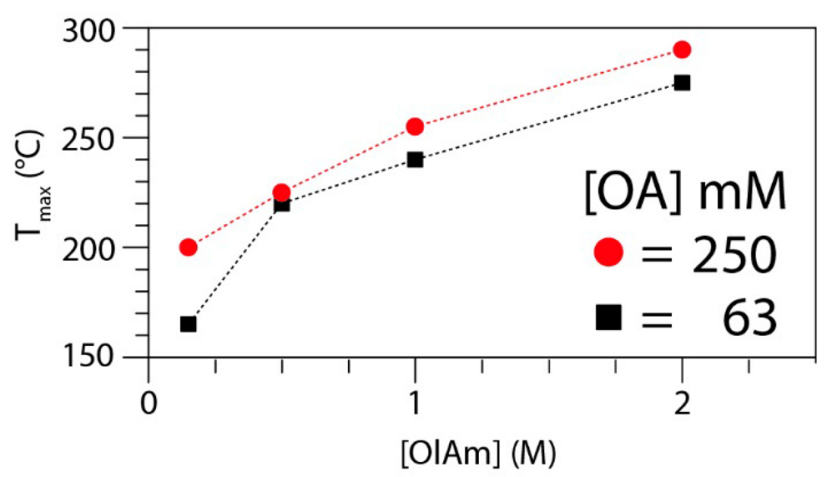

Figure 1. Maximum reaction temperature $T_{\max }$ as a function of ligand concentration; at $T_{\text {max }}, \mathrm{PbBr}_{2}$ precipitates from the reaction medium (33 mM $\mathrm{PbBr}_{2}$ solution in $\mathrm{ODE}$ with [OA] set at a constant of 63 and $250 \mathrm{mM}$ ).

Furthermore, we found that keeping the concentration of ligands close to the minimum amount needed to solubilize the $\mathrm{PbBr}_{2}$ enabled the synthesis of nanocubes with narrow size distributions (Figure 2a).

Examples of NCs that we could synthesize with our general scheme are reported in Figure 2: 4.0 to $7.6 \mathrm{~nm}$ cubes $(\sigma=8-$ $13 \%)$ were prepared at $120-150{ }^{\circ} \mathrm{C}$ in the presence of $0.25 \mathrm{M}$ OlAm and $25 \mathrm{mM}$ OA. Larger cubes were obtained by increasing the reaction temperature, which also required increasing the concentration of ligands. For instance, $16.4 \mathrm{~nm}$ cubes $(\sigma=15 \%)$ were obtained at $220{ }^{\circ} \mathrm{C}$. Syntheses carried out at $240{ }^{\circ} \mathrm{C}([\mathrm{OlAm}]=[\mathrm{OA}]=1.5 \mathrm{M})$ delivered even larger nanocubes (beyond $100 \mathrm{~nm}$ ), but they had a very broad size distribution (see Table 1 and Figure S2 of the SI). All nanocubes synthesized with our approach had crystal structures that matched the orthorhombic $\mathrm{CsPbBr}_{3}$ phase (Figure 2d), narrow photoluminescence (PL) peaks (full-widths at halfmaximum of $70-100 \mathrm{meV}$ for weakly confined samples, $140-$ $170 \mathrm{meV}$ for strongly confined samples, which is in agreement with other reports), ${ }^{4,18}$ and high PL quantum yields (Table 1).

Our strategy of synthesizing $\mathrm{CsPbBr}_{3}$ nanocubes by using the minimum amount of ligands needed to solubilize $\mathrm{PbBr}_{2}$ also suppressed Ostwald ripening, which normally leads to the fast defocusing of NC size within seconds after the injection of $\mathrm{Cs}^{+}$. The suppression of Ostwald ripening was clear, since nearly monodisperse NCs could even be obtained when the reaction was allowed to cool without an ice bath, i.e., solely by removing the heating mantle, a procedure that normally requires at least $10 \mathrm{~min}$ for the flask to cool down (see Figure 2f). Furthermore, under such reduced concentrations of ligands, the formation of nanoplatelets was never observed, regardless of the reaction temperature. Rather, the amount of NPLs increased when the concentration of oleic acid in the reaction mixture was increased (see Figure S3). Following this observation, pure, square-shaped (ca. $10 \times 10 \mathrm{~nm}, 2.4 \mathrm{~nm}$ thick) $\mathrm{CsPbBr}_{3}$ NPLs were prepared at $140{ }^{\circ} \mathrm{C}$ by increasing the concentration of oleic acid in the reaction mixture (from $25 \mathrm{mM}$ to $0.5 \mathrm{M}$ ) as well as in the injection solution (see Figure S4). The thickness of the NPLs could be reduced by decreasing the amount of $\mathrm{Cs}^{+}$ injected (while keeping the amount of OA constant, see Table S2), as is evidenced by the absorbance and PL spectra of the samples (Figure 2b,c, bottom 3). Similar to the findings in previous reports, ${ }^{17,19}$ the NPLs exhibited narrow PL peaks (Figure 2c) and their structural anisotropy was evidenced by strong $(h k 0)$ reflections in their XRD patterns (Figure $2 \mathrm{~d}$; see 

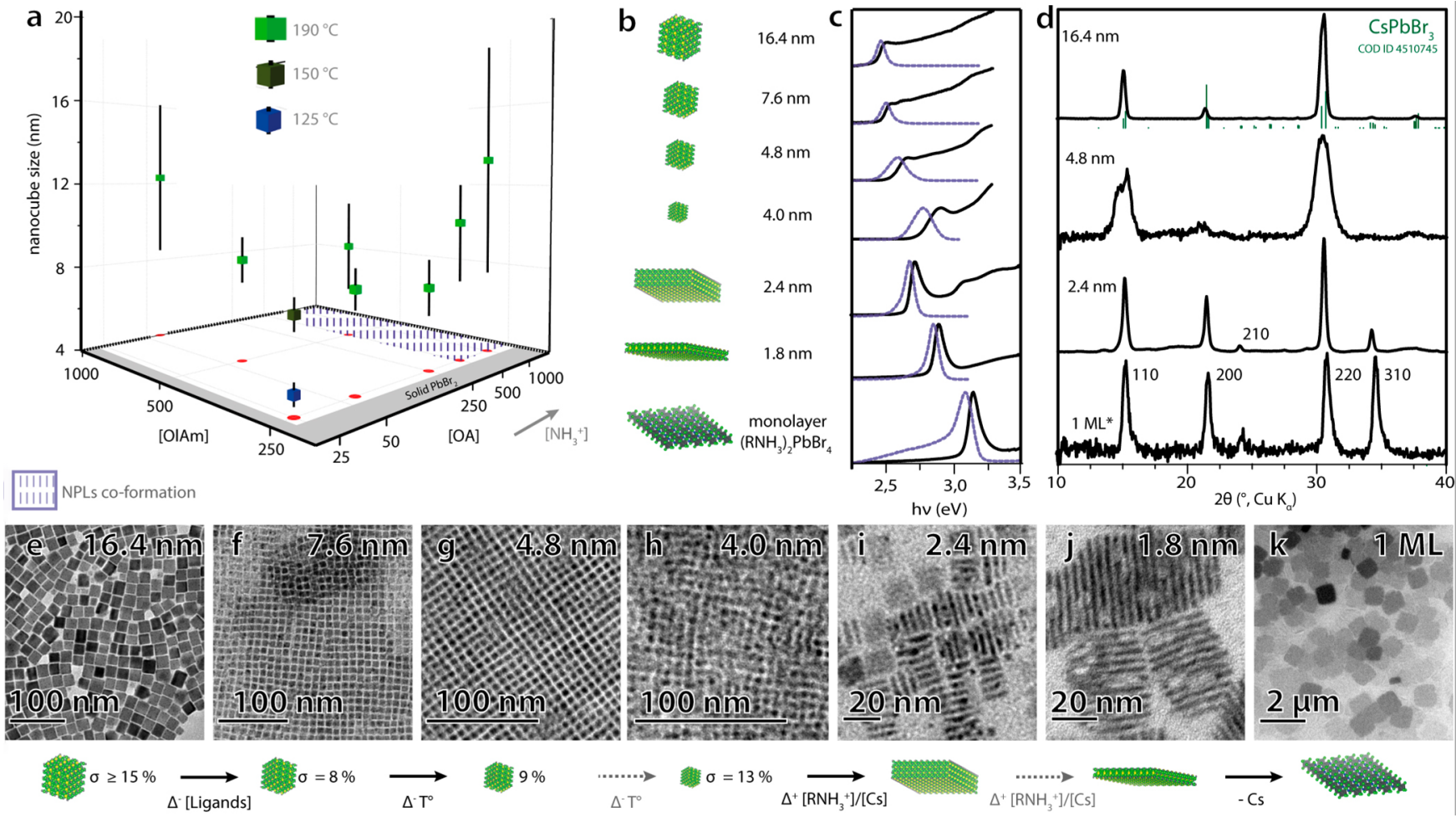

$9 \% \quad \Delta \cdot T^{\circ}$
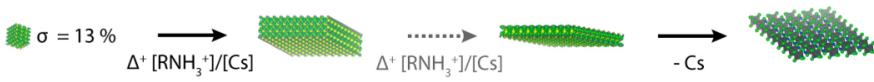

Figure 2. (a) Sizes of $\mathrm{CsPbBr}_{3}$ nanocubes synthesized using various concentrations of oleylamine (OlAm) and oleic acid (OA) and different reaction temperatures (size distributions are represented as vertical bars across each symbol). (b) Image illustrating the range of monodisperse $\mathrm{CsPBr}_{3}$ nanocubes, nanoplatelets, and nanosheets synthesized in this work using only OlAm and OA as ligands. (c) Absorbance (black solid line) and photoluminescence (blue dashed line) spectra. (d) XRD patterns and (e-k) TEM images of the depicted samples.

Table 1. Synthesis Conditions Used To Prepare the $\mathrm{CsPbr}_{3}$ Nanocubes and Their Photoluminescence (PL) Properties ${ }^{a}$

\begin{tabular}{|c|c|c|c|c|c|c|c|c|}
\hline \multicolumn{2}{|c|}{ NC size } & \multicolumn{3}{|c|}{ reaction conditions } & \multicolumn{4}{|c|}{ photoluminescence (PL) } \\
\hline$M(\mathrm{~nm})$ & $\sigma(\%)$ & $T\left({ }^{\circ} \mathrm{C}\right)$ & {$[\mathrm{OlAm}](\mathrm{mM})$} & {$[\mathrm{OA}](\mathrm{mM})$} & $h \nu(\mathrm{eV})$ & fwhm $(\mathrm{meV})$ & $\phi(\%)$ & $\tau(\mathrm{ns})$ \\
\hline$>100$ & $\mathrm{n} / \mathrm{a}$ & 240 & 3500 & 3500 & & & & \\
\hline 16.4 & 15 & 220 & 500 & 500 & 2.39 & 69 & 30 & 45.1 \\
\hline 8.7 & 26 & 190 & 500 & 500 & 2.41 & 83 & & \\
\hline 13.2 & 41 & 190 & 250 & 1000 & 2.41 & 103 & & \\
\hline 10.3 & 26 & 190 & 250 & 500 & 2.41 & 84 & & \\
\hline 7.6 & 16 & 190 & 250 & 250 & 2.41 & 80 & & \\
\hline 8.1 & 10 & 190 & 250 & 63 & 2.42 & 75 & & \\
\hline 8.6 & 12 & 190 & 500 & 63 & 2.41 & 74 & & \\
\hline 12.3 & 31 & 190 & 1000 & 63 & 2.41 & 83 & & \\
\hline 7.6 & 8 & 150 & 250 & 25 & 2.43 & 79 & 49 & 7.6 \\
\hline 4.8 & 9 & 125 & 250 & 25 & 2.53 & 136 & 27 & 5.2 \\
\hline 4.0 & 13 & 120 & 250 & 25 & 2.60 & 170 & 24 & 3.5 \\
\hline
\end{tabular}

${ }^{a}$ Key: $\mu$, mean size; $\sigma$, standard deviation; $T$, synthesis temperature; $h \nu$, photon energy at PL maximum; FWHM, PL full width at half maximum; $\varphi$, PL quantum yield; $\tau$, PL lifetime; -, data not collected. All syntheses were immediately quenched upon injection of $0.5 \mathrm{~mL}$ of Cs-oleate (0.15 M, in 1-ocatadecene) except the $>100 \mathrm{~nm}$ sample where $2 \mathrm{~mL}$ of Cs-precursor was used and the growth time was increased to $60 \mathrm{~s}$.

Figure S5 for a representative high-resolution transmission electron microscopy image).

Interestingly, when $\mathrm{Cs}^{+}$ions were not included in the injection solution, a white precipitate formed upon cooling. This precipitate consisted of micron-sized nanosheets whose crystal phase could be assigned to that of a hybrid organicinorganic two-dimensional perovskite slab with the formula $\left(\mathrm{RNH}_{3}\right)_{2} \mathrm{PbBr}_{4}$, in which $\mathrm{RNH}_{3}$ denotes the oleylammonium ion (the corresponding XRD pattern is the first one from the bottom in Figure 2d). Its PL spectrum (Figure 2c, bottom row) is characterized by a peak at $3.09 \mathrm{eV}$, with a long tail to lower energies, which is in agreement with previous reports for twodimensional perovskites. ${ }^{20,21}$

We have also observed that by increasing the concentrations of both oleic acid and oleylamine in the reaction mixture, we could synthesize $\mathrm{NCs}$ in the lead-poor hexagonal $\mathrm{Cs}_{4} \mathrm{PbBr}_{6}$ phase (Figure $3 \mathrm{c}-\mathrm{e}$ ), which is a nonluminescent insulator characterized by a strong and narrow absorption band resembling that of individual $\left[\mathrm{PbBr}_{6}\right]^{4-}$ clusters (Figure $3 a, b) .{ }^{22,23}$ A similar result was observed by treating $\mathrm{CsPbBr}_{3}$ $\mathrm{NCs}$ with an excess of these same ligands (OA and OlAm), as their phase changed to $\mathrm{Cs}_{4} \mathrm{PbBr}_{6}$. In this case, $8 \mathrm{~nm}$ cube- 


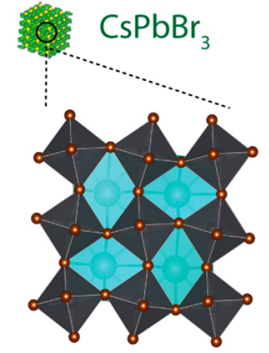

O [100]

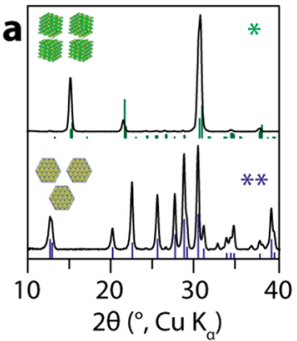

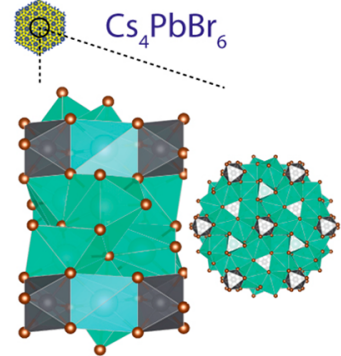

( [001]

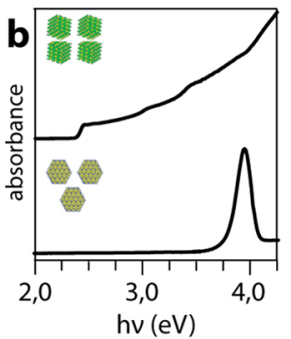

Synthesis
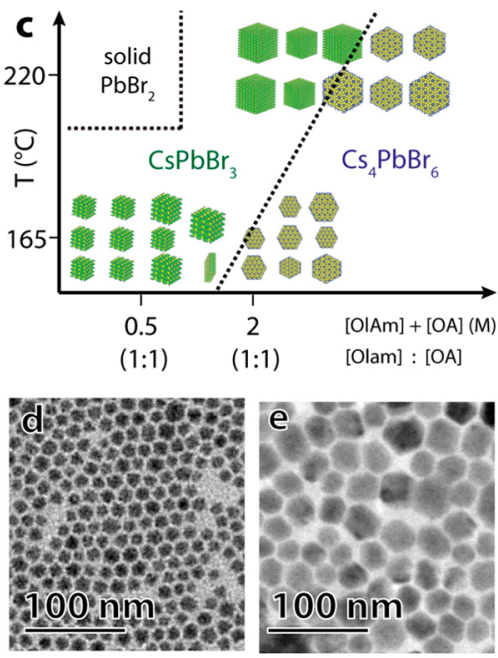

$165^{\circ} \mathrm{C}$

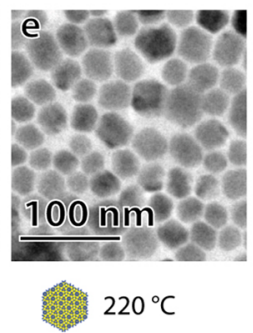

Transformation

f
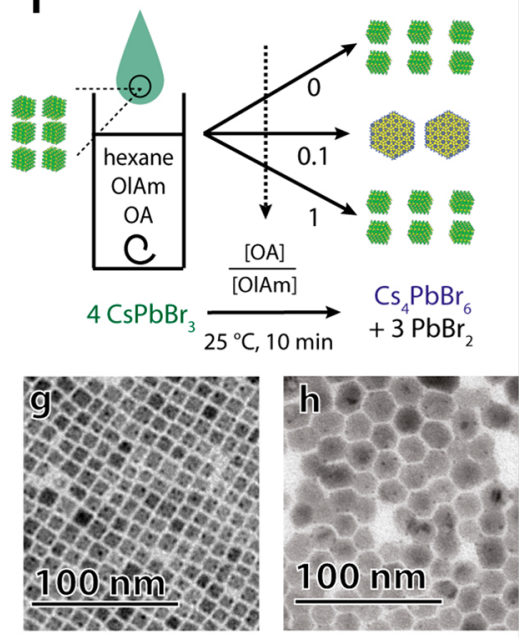

能能

Figure 3. Ligand-mediated synthetic and postsynthetic phase control of cesium lead bromide NCs $(\mathrm{OlAm}=$ oleylamine, $\mathrm{OA}=$ oleic acid $)$. $(\mathrm{a})$ Typical XRD patterns and (b) absorbance spectra of $\mathrm{CsPbBr}_{3}$ and $\mathrm{Cs}_{4} \mathrm{PbBr}_{6} \mathrm{NCs}$ (*COD 4510745; **ICSD 98-009-7851). (c) Scheme depicting how the interplay between the reaction temperature and ligand concentration affects the crystal structure and, hence, the composition of the obtained NCs. TEM image of $\mathrm{Cs}_{4} \mathrm{PbBr}_{6} \mathrm{NCs}$ synthesized at (d) $165{ }^{\circ} \mathrm{C}$ and (e) $220{ }^{\circ} \mathrm{C}$. (f) Scheme depicting the liganddriven transformation of $\mathrm{CsPbBr}_{3}$ NCs into $\mathrm{Cs}_{4} \mathrm{PbBr}_{6}$ NCs: in short, $100 \mu \mathrm{L}$ of a dispersion containing $\mathrm{CsPbBr}_{3}$ nanocubes was added to a solution $(1.0 \mathrm{~mL})$, kept under stirring, that contained a fixed concentration of OlAm $(0.4 \mathrm{M})$ and various concentrations of $\mathrm{OA}$ and solvent (hexane). This transformation was followed by spectroscopy analysis, and the transformation rate was found to be modulated by the [OA]/ $[\mathrm{OlAm}]$ ratio, as shown in the figure above. For the sake of simplicity, only the state of the systems at $t=10 \mathrm{~min}$ is shown. TEM images of NCs $(\mathrm{g})$ before and $(\mathrm{h})$ after the transformation.
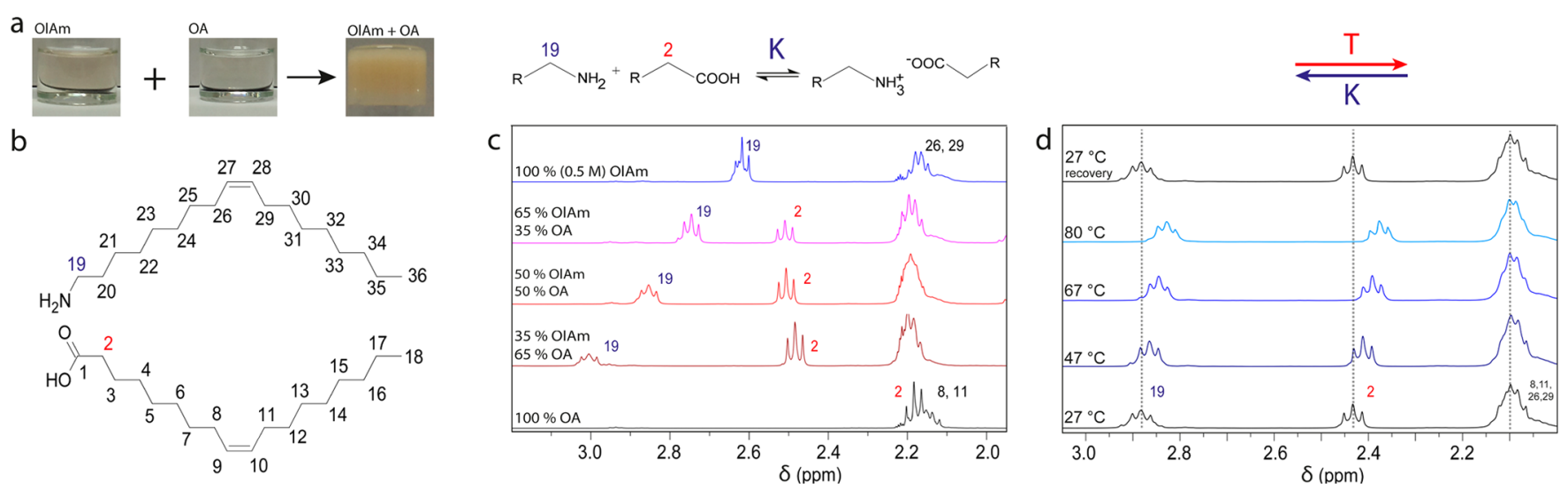

Figure 4. (a) Photographs illustrating the formation of the oleylammonium oleate salt. (b) Structure of oleylamine (OIAm) and oleic acid (OA). (c) Selected regions of the ${ }^{1} \mathrm{H}$ NMR spectra of OlAm and OA solutions in toluene- $d_{8}$. (d) ${ }^{1} \mathrm{H}$ NMR spectra of a mixture of OlAm and $\mathrm{OA}$ in toluene- $d_{8}$, recorded at different temperatures in the range of 27 to $80{ }^{\circ} \mathrm{C}$ (upfield shifts are observed for the $\alpha$ - $\mathrm{CH}_{2}{ }^{1} \mathrm{H} \mathrm{NMR}$ resonances 19 and 2, while all the other signals, e.g., 8, 11, 26, and 29, remain unchanged). All resonances identified are in agreement with previous works. 23,33

shaped $\mathrm{CsPbBr}_{3}$ NCs could be transformed to ca. $20 \mathrm{~nm}$ hexagonal $\mathrm{Cs}_{4} \mathrm{PbBr}_{6}$ NCs. This increase in $\mathrm{NC}$ size is in agreement with the two-step dissolution-recrystallization mechanism that is reported in previous works. ${ }^{24,25}$ Interestingly, the rate of this transformation was found to be dictated by the ratio between OA and OlAm and will be discussed in the next section.

\section{DISCUSSION}

Acid-base Equilibrium. This section discusses the acidbase chemistry of OlAm and OA mixtures in nonpolar solvents and how the size, shape, and phase control of cesium lead bromide NCs are regulated. It is known that, in aprotic solvents, partially substituted nitrogen bases and carboxylic acids can form hydrogen bonded ionic salts. ${ }^{26}$ The first hint of the formation of ammonium carboxylate salts can be observed by simply mixing OlAm (base, $\mathrm{B}$ ) and $\mathrm{OA}(\mathrm{AH})$ in a 1:1 molar ratio at room temperature. This generates heat and leads to the formation of a gel (Figure 4a), indicating that the ions mainly associate in their salt form under these conditions.

$$
\mathrm{B}+\mathrm{H}-\mathrm{A} \leftrightarrow \mathrm{BH}^{+} \cdots \mathrm{A}^{-}
$$

To confirm the formation of this ammonium carboxylate, nuclear magnetic resonance (NMR) analysis was performed on 
OlAm and OA mixtures in toluene- $d_{8}$ (Figure $4 \mathrm{c}$ ). In the presence of an excess of OA, the $\alpha-\mathrm{CH}_{2}$ resonances of OlAm (19) exhibit a downfield shift in the ${ }^{1} \mathrm{H}$ NMR spectrum, which occurs as a consequence of the nitrogen protonation, while the $\alpha-\mathrm{CH}_{2}$ resonances of OA (2) show a downfield shift with the deprotonation of the carboxylic acid (Figure 4b). Opposite shifts are observed in the ${ }^{13} \mathrm{C}$ NMR spectrum; i.e., upfield shifts occur for the $\alpha-\mathrm{CH}_{2}$ carbon of both the OlAm and the OA as a result of the protonation of the adjacent functional group (Figure S7.2). In the SI, section S6, we report the complete assignment of the components in a model mixture of $0.5 \mathrm{M}$ OlAm and $1 \mathrm{M} \mathrm{OA}$ in toluene- $d_{8}$. The $\alpha-\mathrm{CH}_{2}$ resonances of OlAm (19) and those of OA (2) were unambiguously identified by the ${ }^{1} \mathrm{H}-{ }^{13} \mathrm{C}-\mathrm{HMBC}$ NMR spectrum (Figure S6.1d).

As previously mentioned, the formation of the ammonium carboxylate is exothermic, which implies, on the basis of Le Chatelier's principle, that the equilibrium will shift toward the reactants when the temperature is increased. This was also confirmed by ${ }^{1} \mathrm{H}$ NMR spectroscopy. Notably, the $\alpha-\mathrm{CH}_{2}{ }^{1} \mathrm{H}$ NMR resonances of the protonated OlAm (19) and those of OA (2) shifted upfield when the temperature was increased, which confirms the deprotonation of the oleylammonium ions and the protonation of the carboxylic acid (Figure 4d). These facts have important implications in the colloidal synthesis and phase stability of $\mathrm{Cs} \mathrm{PbBr}_{3} \mathrm{NCs}$, as will be outlined in the next section.

Role of Ligands in Size and Shape Control. First, we reemphasize the fact that both ligands are necessary to solubilize solid $\mathrm{PbBr}_{2}$, which highlights the active role of both the ammonium and carboxylate species in this process. In this system, $\mathrm{PbBr}_{2}$ exhibits an inverse solubility behavior (see Figure 1) that is related to the decrease in the concentration of ammonium and carboxylate species when the temperature is increased, as has been previously demonstrated. In addition, we found that this binary ligand mixture can also dissolve $\mathrm{CsPbB}$ NCs (Figure $3 \mathrm{f}-\mathrm{h}$ ). This phenomenon is in agreement with the strong Ostwald ripening effects that were observed during the NC growth. ${ }^{14}$ Accordingly, we found that Ostwald ripening could be suppressed by reducing the concentration of ligands, as is evidenced by the narrower size distributions of the NCs that were obtained following our strategy (Figure 2).

Second, it is now well established that aliphatic ammonium $\left(\mathrm{RNH}_{3}^{+}\right)$ions can compete with $\mathrm{Cs}^{+}$ions for lattice sites and promote the formation of hybrid layered structures with the general formula $\left[\mathrm{RNH}_{3}\right]_{2}[\mathrm{CsPbBr}]_{n-1} \mathrm{PbBr}_{4}$ (in which $\mathrm{n}$ denotes the number of $\mathrm{PbBr}_{6}$ octahedra along the thickness). ${ }^{27,28}$ Indeed, our results in terms of shape control showed that NPLs could be obtained by increasing the $\left[\mathrm{RNH}_{3}{ }^{+}\right] /\left[\mathrm{Cs}^{+}\right]$ ratio. Following this concept, and accounting the temperature dependence of $\left[\mathrm{RNH}_{3}^{+}\right]$, pure NPL samples could be synthesized even at high temperatures $\left(190{ }^{\circ} \mathrm{C}\right)$ by simply adjusting the $\left[\mathrm{Cs}^{+}\right]$accordingly (see Table S2 and Figure S7). Moreover, such two-dimensional hybrid systems could also be synthesized in the presence of other Brønsted acids. Notably, when the acid strength increased (benzylsulfonic $\gg$ hexanoic $>$ oleic acid), lower amounts of acid were needed to begin forming such structures (Figure S8).

Mechanistic Insights. The solubility of $\mathrm{PbBr}_{2}$ in this system can be interpreted on the basis of the dissociation of the oleylammonium oleate salt followed by the solvation of $\mathrm{PbBr}_{2}$. In addition to thermal effects, the dissociation of acid base salts in aprotic solvents can also occur via homoconjugation

$$
\mathrm{BH}^{+} \cdots \mathrm{A}^{-}+\mathrm{B} \leftrightarrow\left(\mathrm{BH}^{+} \cdots \mathrm{B}\right) \mathrm{A}^{-}
$$

a mechanism that typically shows high equilibrium constants for amine-based systems. ${ }^{26,29-31}$

The interpretation of the oleylammonium oleate salt as an inactive species is supported by our results in terms of phase transformation (Figure $3 \mathrm{f}-\mathrm{h}$ ). In short, the transformation of $\mathrm{Cs} \mathrm{PbBr}_{3}$ into $\mathrm{Cs}_{4} \mathrm{PbBr}_{6}$ is driven by the ability of the ligand mixture to solubilize $\mathrm{PbBr}_{2}$, which is the byproduct of this transformation. Our results demonstrate that the kinetics of this reaction is modulated by the relative amounts of acid and base (Figure 3f). The transformation does not proceed in the absence of acid but is found to be completed in a few minutes when the amount of acid is ca. $10 \% \mathrm{~mol}$ of that of the amine. However, increasing the concentration of acid any further slows down the transformation until it does not proceed again. Therefore, at room temperature, a 1:1 molar mixture of oleylamine and oleic acid, which mainly exist in salt form, gelates in the absence of a solvent. However, when an excess of base is present, it can dissociate into ionic species which, in turn, can dissolve $\mathrm{PbBr}_{2}$ and drive the transformation.

Furthermore, we found that $\mathrm{PbBr}_{2}$ (1 equiv) can be dissolved at mild temperatures (ca. $80-100{ }^{\circ} \mathrm{C}$ ) in the presence of a minimum amount of 0.5 equiv of stearic acid and an excess of amine ligands (4-5 equiv) (here, octadecylamine and stearic acid were used due to their high purity), which is likely a result of the partial dissociation of $\mathrm{PbBr}_{2}$ :

$$
2 \mathrm{PbBr}_{2}+\mathrm{BH}^{+} \cdots \mathrm{A}^{-}+x \mathrm{~B} \rightarrow \mathrm{PbBr}_{3}{ }^{-} \cdot \mathrm{BH}^{+}+\mathrm{PbBr}^{+} \cdot \mathrm{A}^{-}+x \mathrm{~B}
$$

Note that the solubility of $\mathrm{PbBr}_{2}$ in polar aprotic solvents increases in the presence of methylammonium bromide, and recent computational studies have related this phenomenon to the formation of $\mathrm{PbBr}_{3}-$ methyammonium species with an increased stability. ${ }^{9}$

Our results, in terms of phase control (Figure 3c-e), demonstrate that the NCs tend to crystallize in the lead-poor $\mathrm{Cs}_{4} \mathrm{PbBr}_{6}$ phase when both ligands are present in large excess. Under such conditions, a full dissociation of the lead salt into lead oleate and oleylammonium bromide is likely to occur:

$\mathrm{PbBr}_{2}+2\left(\mathrm{BH}^{+} \cdots \mathrm{B}\right) \mathrm{A}^{-} \rightarrow \mathrm{Pb} \cdot \mathrm{A}_{2}+2\left(\mathrm{BH}^{+} \cdots \mathrm{Br}^{-}\right)+2 \mathrm{~B}$

This system allows a greater degree of freedom than the one described by eq 2 with respect to possible outcomes upon the injection of $\mathrm{Cs}^{+}$ions. For instance, the reaction of an alkylammonium bromide with $\mathrm{Cs}^{+}$in the same ligand mixture results in the formation of CsBr NCs (Figure S9). The two extreme situations illustrated in eqs 3 and 4 can thus explain our results in terms of ligand-mediated phase control. Again, given the temperature profile of the acid base-equilibrium, a system described by eq 4 should shift to the one described by eq 3 when the temperature is increased. Indeed, we could find a ligand concentration that yields $\mathrm{Cs}_{4} \mathrm{PbBr}_{6} \mathrm{NCs}$ at low temperatures $\left(165^{\circ} \mathrm{C}\right)$, and $\mathrm{CsPbBr}_{3} \mathrm{NCs}$ at high temperatures $\left(220{ }^{\circ} \mathrm{C}\right.$, Figure $\left.2 \mathrm{c}\right)$.

Extension to Other Halide Systems. Most of the findings reported herein were also found to be valid for the $\mathrm{Cl}$ and $\mathrm{I}$ systems. For instance, $\mathrm{PbCl}_{2}$ and $\mathrm{PbI}_{2}$ also exhibit inverse solubility in this binary ligand system, and the reprecipitation temperatures follow a similar trend to that found for $\mathrm{PbBr}_{2}$ (see Figure S11). Furthermore, $\mathrm{CsPbX}_{3} \mathrm{NCs}, \mathrm{Cs}_{4} \mathrm{PbX}_{6} \mathrm{NCs}$, and $\left(\mathrm{RNH}_{3}\right)_{2} \mathrm{PbX}_{4}$ nanosheets $(\mathrm{X}=\mathrm{Cl}, \mathrm{I})$ could also be synthesized following the same strategies presented here for the phase and 
shape control of bromides (see Figures S12 and S13). Unfortunately, for the $\mathrm{Cl}$ and I cases, due to the poorer solubility of $\mathrm{PbCl}_{2}$ and the lower reprecipitation temperature of $\mathrm{PbI}_{2}$ in this binary ligand system, we could not reproduce the low ligand concentration conditions which had enabled us to synthesize $\mathrm{CsPbBr}_{3}$ nanocubes with narrow size distributions.

\section{CONCLUSION}

In this work, we have shown how the acid-base interactions within a ligand system that consists of aliphatic primary amines and carboxylic acids affect the synthesis and transformation of cesium lead bromide nanocrystals in nonpolar solvents. This allowed us not only to achieve precise control over the size, shape, and phase of the nanocrystals but also to understand the solubility behavior of $\mathrm{PbBr}_{2}$ in this system. In addition, many of the findings reported herein were also found to be valid for the $\mathrm{Cl}$ and I systems. Furthermore, one should be able to extend these observations to nonprotic polar solvents such as dimethylformamide, a commonly used solvent in the synthesis of lead halide perovskites, which is known to self-decompose into dimethylamine and formic acid ${ }^{12}$ and is able to sustain the growth of perovskite single crystals via inverse solubility. Finally, the fact that this binary ligand system is able to dissolve $\mathrm{CsPbX}_{3}$ nanocrystals and cannot provide good surface passivation $^{34}$ should prompt further studies into other ligand systems.

\section{METHODS}

Materials. $\mathrm{PbCl}_{2}(\geq 99 \%)$ was purchased from Strem Chemicals. $\mathrm{PbBr}_{2}$ ( $\left.\geq 98 \%\right), \mathrm{PbI}_{2}$ ( $\left.\geq 99.999 \%\right)$, oleylamine (70\%, OlAm), octadecylamine $(\geq 98 \%)$, oleic acid $(90 \%$, OA), stearic acid $(95 \%)$, hexanoic acid ( $\geq 99 \%$, HA), benzenesulfonic acid ( $\geq 94 \%$, BSA), didodecyldimethylammonium bromide ( $\geq 98 \%$, DDAB), 1-octadecene (90\%), toluene ( $\geq 99.7 \%)$, toluene- $d_{8}$ (99 atom. \% D), hexane ( $\geq 95 \%$ ), 2-propanol $(\geq 99.8 \%$ ), and methyl acetate (anhydrous, 99.5\%) were purchased from Sigma-Aldrich. All chemicals were used without any further purification unless otherwise stated.

Preparation of Cs-Oleate Precursors. Solution 1. Cs-Oleate in 1-Octadecene $\left(\mathrm{Cs}_{2} \mathrm{CO}_{3} / \mathrm{OA}=26 \% \mathrm{wt}\right)$. $\mathrm{Cs}_{2} \mathrm{CO}_{3}$ was weighed inside a 3-neck round-bottom flask along with oleic acid and 1-octadecene. The mixture was degassed under vacuum at $100{ }^{\circ} \mathrm{C}$ for $2 \mathrm{~h}$ in order to obtain a colorless Cs-oleate solution which was then transferred into the glovebox. Typically, $407 \mathrm{mg} \mathrm{Cs}_{2} \mathrm{Co}_{3}, 1.55 \mathrm{~g} \mathrm{OA}$ and $11.7 \mathrm{~g} \mathrm{ODE}$ were combined to obtain a $0.15 \mathrm{M}$ Cs-oleate solution.

Solution 2. Cs-Oleate in Oleic Acid. $\mathrm{Cs}_{2} \mathrm{CO}_{3}$ was weighed inside a three-neck round-bottom flask along with oleic acid (see Table S1 for concentrations). The mixture was degassed under vacuum at $100{ }^{\circ} \mathrm{C}$ for $2 \mathrm{~h}$ in order to obtain a colorless Cs-oleate solution which was then transferred into the glovebox. Diluted solutions were prepared inside the glovebox by dilution with degassed oleic acid.

Syntheses (General Considerations). All synthetic procedures were undertaken by employing standard Schlenk line techniques assisted by a nitrogen-filled glovebox where lead(II) bromide and the Cs precursors were stored. Syntheses were performed in $25 \mathrm{~mL}$ 3-neck round-bottom flasks equipped with a thermocouple and a magnetic stirrer at $800 \mathrm{rpm}$.

Synthesis of Cube-Shaped $\mathrm{CsPbBr}_{3}$ Nanocrystals (NCs). All NCs were synthesized in $6.0 \mathrm{~mL}$ solutions containing $72 \mathrm{mg}$ of $\mathrm{PbBr}_{2}$ $(0.2 \mathrm{mmol}, 33 \mathrm{mM})$ and various amounts of oleylamine, oleic acid, and 1-octadecene (see Table 1). Mixtures were degassed for $15 \mathrm{~min}$ at 100 ${ }^{\circ} \mathrm{C}$ in order to obtain colorless solutions. Thereafter, the temperature was ramped to the desired value under a dry nitrogen flow and $0.5 \mathrm{~mL}$ of a $0.15 \mathrm{M}$ Cs-oleate solution in 1-octadecene (solution 1, which had previously been heated for $10 \mathrm{~min}$ on a hot-plate set at $200{ }^{\circ} \mathrm{C}$ ) was swiftly injected. Unless otherwise stated, the solutions were immediately cooled after injection with an ice bath and diluted with
$5 \mathrm{~mL}$ of toluene. The dispersions were centrifuged at $2500 \mathrm{rpm}$ (for 3 $\mathrm{min}$ ) and the NCs were redispersed in $2.0 \mathrm{~mL}$ of hexane. 4.0 and 4.8 $\mathrm{nm}$ nanocubes could not be separated by centrifugation alone (even at $14 \mathrm{krpm}$ ). In both these cases, $1 \mathrm{~mL}$ of oleic acid was added to the dispersion (to assist the precipitation process) and the particles were then precipitated by adding methyl acetate until the solution became slightly turbid. The NCs were separated by the same centrifugation and redispersion steps. NCs smaller than $4.0 \mathrm{~nm}$ could also be synthesized (at temperatures under $120{ }^{\circ} \mathrm{C}$ ), but they could not be isolated from solution by centrifugation alone, and the addition of antisolvents led to the formation of nanowires (see Figure S10).

Synthesis of Two-Dimensional $\left[\mathrm{RNH}_{3}\right]_{2}\left[\mathrm{Cs} \mathrm{PbBr}_{3}\right]_{n-1} \mathrm{PbBr}_{4}$ Nanocrystals. The general procedure consisted in performing the nanocube synthesis under a high concentration of oleylammonium species $([\mathrm{OlAm}]=0.25 \mathrm{M}$ and $[\mathrm{OA}]=0.50 \mathrm{M})$ and varying the concentration of $\mathrm{Cs}^{+}$(the smaller the $\left[\mathrm{Cs}^{+}\right]$, the thinner the crystals). Note that in this case the injection solution consisted of $0.5 \mathrm{~mL}$ of a solution of Cs-oleate in oleic acid (solution $2,0 \leq\left[\mathrm{Cs}^{+}\right] \leq 300 \mathrm{mM}$ ) and that the growth times ranged from 1 to $5 \mathrm{~min}$; a complete description of the reaction conditions is provided in Table S1 of the SI. Also note that the $1.7 \mathrm{~nm}$ thick nanoplatelets (NPLs) tend to transform into $2.3 \mathrm{~nm}$ thick NPLs upon dilution, which did not allow us to investigate their photoluminescence quantum yields.

$\mathrm{CsPbBr}_{3}$ to $\mathrm{Cs}_{4} \mathrm{PbBr}_{6}$ Transformation Reactions. $100 \mu \mathrm{L}$ of a nanocube dispersion $([\mathrm{Pb}]=13 \mathrm{mM}$, as measured by ICP) was injected into a stirring hexane solution containing oleylamine $(0.4 \mathrm{M})$ and oleic acid (of various concentrations) with a total volume of 1.0 $\mathrm{mL}$. Aliquots were collected over time in hexane in order to follow their transformation spectroscopically. The transformed NCs were separated by centrifugation and redispersed in hexane or toluene for further analysis.

Inductively Coupled Plasma-Optical Emission Spectroscopy (ICP-OES). The concentration of NC dispersions in $\mathrm{Pb}$ was determined by ICP-OES on a aiCAP 6000 spectrometer (Thermo Scientific). The NC solutions were digested in aqua regia overnight prior to the measurements.

X-ray Diffraction (XRD). Samples were prepared by drop-casting concentrated dispersions onto a zero diffraction silicon substrate. XRD measurements were conducted on a PANalytical Empyrean X-ray diffractometer equipped with a $1.8 \mathrm{~kW} \mathrm{Cu} \mathrm{K} \alpha$ ceramic X-ray tube and PIXcel3D $2 \times 2$ area detector, operating at $45 \mathrm{kV}$ and $40 \mathrm{~mA}$. The diffraction patterns were collected in air at room temperature using parallel-beam (PB) geometry and symmetric reflection mode. Due to preferential orientation, the diffraction pattern of the $\left(\mathrm{RNH}_{3}\right)_{2} \mathrm{PbBr}_{4}$ nanosheets was collected using an in-plane geometry on a Rigaku SmartLab $9 \mathrm{~kW}$ with the X-ray source operated at $40 \mathrm{kV}$ and $150 \mathrm{~mA}$.

Transmission Electron Microscopy (TEM). Bright field TEM images were acquired on a JEOL JEM-1011 microscope (W filament) operating at an accelerating voltage of $100 \mathrm{kV}$. For this purpose, samples were prepared by drop-casting dispersions on carbon-coated 200 mesh copper grids. High-resolution TEM (HRTEM) and highangle annular dark field scanning (HAADF) images were acquired on a JEOL JEM-2200FS microscope, operating at $200 \mathrm{kV}$. The microscope is equipped with a CEOS objective corrector, allowing a resolution below $0.9 \AA$, and an in-column filter ( $\Omega$-type), which was used to increase the contrast in the images by filtering around the elastically transmitted electrons. To obtain a top-view and side-view of the NCs, the samples were drop-cast on ultrathin carbon on holey carbon coated 400 mesh copper grids.

Nuclear Magnetic Resonance. All spectra were acquired on a Bruker Avance III $400 \mathrm{MHz}$ spectrometer, equipped with a Broad Band Inverse probe (BBI). Before each acquisition, automatic routines optimized the matching, tuning, resolution and 90 deg pulse calculation on ${ }^{1} \mathrm{H}$ nucleus.

${ }^{1} \mathrm{H}$ NMR: 16 transients were accumulated, without steady state scans, at $300 \mathrm{~K}$, over a spectral width of $20.55 \mathrm{ppm}$ (offset at 6.175 $\mathrm{ppm}$ ), at a fixed receiver gain (64), using $30 \mathrm{~s}$ of inter pulse delays.

${ }^{1} \mathrm{H}$ NMR at different temperatures (from 300 to $353 \mathrm{~K}$ ): 16 transients were accumulated, with 4 steady state scans, over a spectral 
width of $20.55 \mathrm{ppm}$ (offset at $6.175 \mathrm{ppm}$ ), at a fixed receiver gain (64), using $2.5 \mathrm{~s}$ of relaxation delay.

${ }^{13} \mathrm{C}$ NMR: 10240 transients were accumulated after a 30 degree pulse and 4 steady state scans, at $300 \mathrm{~K}$, over a spectral width of 239 ppm (offset at $100 \mathrm{ppm}$ ), using $2.2 \mathrm{~s}$ of inter pulse delays. The receiver was automatically optimized.

All NMR chemicals shifts were referred to the not deuterated toluene residue peak at $7.09 \mathrm{ppm}$ and $129.24 \mathrm{ppm}$ at ${ }^{1} \mathrm{H}$ - and ${ }^{13} \mathrm{C}$ NMR, respectively.

Steady-State UV-vis Extinction and Photoluminescence Spectroscopy. Optical extinction and photoluminescence spectra of hexane dispersions were recorded in quartz cuvettes with a $1 \mathrm{~cm}$ path-length, employing a Varian Cary $300 \mathrm{UV}$-vis spectrophotometer and a Varian Cary Eclipse fluorescence spectrophotometer, respectively.

Photoluminescence Quantum Yields and Time-Correlated Single-Photon Counting. The time-resolved photoluminescence spectra were measured using an Edinburgh Instruments FLS920 spectrofluorometer. The PL decay traces were recorded by exciting the samples at $405 \mathrm{~nm}$ using a 50 ps laser diode. The data were collected at the PL peak position with an emission bandwidth of $10 \mathrm{~nm}$. The photoluminescence quantum efficiencies were measured using the same instrument with an integrating sphere, exciting the nanocrystal solution at $400 \mathrm{~nm}$. The optical density of the nanocrystal solution was 0.1 at $400 \mathrm{~nm}$.

\section{ASSOCIATED CONTENT}

\section{S Supporting Information}

The Supporting Information is available free of charge on the ACS Publications website at DOI: 10.1021/acsnano.7b08357.

Synthetic details, control, and synthesis of CsBr NCs; additional structural characterization of NPLs (highresolution transmission electron microscopy); additional NMR characterization of OlAm/OA solutions $\left({ }^{13} \mathrm{C}\right.$, ${ }^{1} \mathrm{H}-{ }^{13} \mathrm{C}-\mathrm{HSQC}$, and ${ }^{1} \mathrm{H}-{ }^{13} \mathrm{C}-\mathrm{HMBC}$ spectra) (PDF)

\section{AUTHOR INFORMATION}

\section{Corresponding Author}

*E-mail: liberato.manna@iit.it.

\section{ORCID $\odot$}

Iwan Moreels: 0000-0003-3998-7618

Liberato Manna: 0000-0003-4386-7985

Notes

The authors declare no competing financial interest.

\section{ACKNOWLEDGMENTS}

We acknowledge Dr. Ahmed Abdelhady for stimulating discussions, and Ms. Emma de Cecco for proofreading the manuscript. The research leading to these results has received funding from the FP7 under Grant Agreement No. 614897 (ERC Consolidator Grant "TRANS-NANO”).

\section{REFERENCES}

(1) Kojima, A.; Teshima, K.; Shirai, Y.; Miyasaka, T. Organometal Halide Perovskites as Visible- Light Sensitizers for Photovoltaic Cells. J. Am. Chem. Soc. 2009, 131, 6050-6051.

(2) Park, N.-G.; Grätzel, M.; Miyasaka, T.; Zhu, K.; Emery, K. Towards Stable and Commercially Available Perovskite Solar Cells. Nat. Energy 2016, 1, 16152.

(3) Yang, W. S.; Park, B.-W.; Jung, E. H.; Jeon, N. J.; Kim, Y. C.; Lee, D. U.; Shin, S. S.; Seo, J.; Kim, E. K.; Noh, J. H.; Seok, S., Il. Iodide Management in Formamidinium-Lead-Halide-based Perovskite Layers for Efficient Solar Cells. Science 2017, 356, 1376-1379.

(4) Protesescu, L.; Yakunin, S.; Bodnarchuk, M. I.; Krieg, F.; Caputo, R.; Hendon, C. H.; Yang, R. X.; Walsh, A.; Kovalenko, M. V.
Nanocrystals of Cesium Lead Halide Perovskites $\left(\mathrm{CsPbX}_{3}, \mathrm{X}=\mathrm{Cl}, \mathrm{Br}\right.$ and I): Novel Optoelectronic Materials Showing Bright Emission with Wide Color Gamut. Nano Lett. 2015, 15, 3692-3696.

(5) Nedelcu, G.; Protesescu, L.; Yakunin, S.; Bodnarchuk, M. I.; Grotevent, M. J.; Kovalenko, M. V. Fast Anion-Exchange in Highly Luminescent Nanocrystals of Cesium Lead Halide Perovskites $(\mathrm{CsPbX}, \mathrm{X}=\mathrm{Cl}, \mathrm{Br}, \mathrm{I})$. Nano Lett. 2015, 15, 5635-5640.

(6) Sutherland, B. R.; Sargent, E. H. Perovskite Photonic Sources. Nat. Photonics 2016, 10, 295-302.

(7) Bush, K. A.; Palmstrom, A. F.; Yu, Z. J.; Boccard, M.; Cheacharoen, R.; Mailoa, J. P.; McMeekin, D. P.; Hoye, R. L. Z.; Bailie, C. D.; Leijtens, T.; Peters, I. M.; Minichetti, M. C.; Rolston, N.; Prasanna, R.; Sofia, S.; Harwood, D.; Ma, W.; Moghadam, F.; Snaith, H. J.; et al. 23.6\%-Efficient Monolithic Perovskite/Silicon Tandem Solar Cells With Improved Stability. Nat. Energy 2017, 2, 17009.

(8) Pan, A.; He, B.; Fan, X.; Liu, Z.; Urban, J. J.; Alivisatos, A. P.; He, L.; Liu, Y. Insight into the Ligand-Mediated Synthesis of Colloidal Cs $\mathrm{PbBr}_{3}$ Perovskite Nanocrystals: The Role of Organic Acid, Base, and Cesium Precursors. ACS Nano 2016, 10, 7943-7954.

(9) Stevenson, J.; Sorenson, B.; Subramaniam, V. H.; Raiford, J.; Khlyabich, P. P.; Loo, Y. L.; Clancy, P. Mayer Bond Order as a Metric of Complexation Effectiveness in Lead Halide Perovskite Solutions. Chem. Mater. 2017, 29, 2435-2444.

(10) Nayak, P. K.; Moore, D. T.; Wenger, B.; Nayak, S.; Haghighirad, A. A.; Fineberg, A.; Noel, N. K.; Reid, O. G.; Rumbles, G.; Kukura, P.; Vincent, K. A.; Snaith, H. J. Halide Perovskite Crystals. Nat. Commun. 2016, 7, 13303.

(11) Noel, N. K.; Habisreutinger, S. N.; Wenger, B.; Klug, M. T.; Hörantner, M. T.; Johnston, M. B.; Nicholas, R. J.; Moore, D. T.; Snaith, H. J. A Low Viscosity, Low Boiling Point, Clean Solvent System for the Rapid Crystallisation of Highly Specular Perovskite Films. Energy Environ. Sci. 2017, 10, 145-152.

(12) Noel, N. K.; Congiu, M.; Ramadan, A. J.; Fearn, S.; Mcmeekin, D. P.; Patel, J. B.; Johnston, M. B.; Wenger, B.; Snaith, H. J. Joule Unveiling the Underlying Influence of $\mathrm{pH}$ on the Crystallization of Hybrid Perovskite Unveiling the Underlying Influence of $\mathrm{pH}$ on the Crystallization of Hybrid Perovskite Materials, Delivering Low Voltage Loss Photovoltaics. Joule 2017, 1, 328-343.

(13) Sun, S.; Yuan, D.; Xu, Y.; Wang, A.; Deng, Z. Ligand-Mediated Synthesis of Shape-Controlled Cesium Lead Halide Perovskite Nanocrystals via Reprecipitation Process at Room Temperature. ACS Nano 2016, 10, 3648-3657.

(14) Koolyk, M.; Amgar, D.; Aharon, S.; Etgar, L. Kinetics of Cesium Lead Halide Perovskite Nanoparticle Growth; Focusing and deFocusing of Size Distribution. Nanoscale 2016, 8, 6403-6409.

(15) Bekenstein, Y.; Koscher, B. A.; Eaton, S. W.; Yang, P.; Alivisatos, A. P. Highly Luminescent Colloidal Nanoplates of Perovskite Cesium Lead Halide and Their Oriented Assemblies. J. Am. Chem. Soc. 2015, 137, 16008-16011.

(16) Sichert, J. A.; Tong, Y.; Mutz, N.; Vollmer, M.; Fischer, S.; Milowska, K. Z.; García Cortadella, R.; Nickel, B.; Cardenas-Daw, C.; Stolarczyk, J. K.; Urban, A. S.; Feldmann, J. Quantum Size Effect in Organometal Halide Perovskite Nanoplatelets. Nano Lett. 2015, 15, $6521-6527$.

(17) Akkerman, Q. A.; Motti, S. G.; Srimath Kandada, A. R.; Mosconi, E.; D’Innocenzo, V.; Bertoni, G.; Marras, S.; Kamino, B. A.; Miranda, L.; De Angelis, F.; Petrozza, A.; Prato, M.; Manna, L. Solution Synthesis Approach to Colloidal Cesium Lead Halide Perovskite Nanoplatelets with Monolayer-Level Thickness Control. J. Am. Chem. Soc. 2016, 138, 1010-1016.

(18) Brennan, M. C.; Zinna, J.; Kuno, M. Existence of a SizeDependent Stokes Shift in $\mathrm{CsPbBr}_{3}$ Perovskite Nanocrystals. ACS Energy Lett. 2017, 2, 1487-1488.

(19) Shamsi, J.; Dang, Z.; Bianchini, P.; Canale, C.; Di Stasio, F.; Brescia, R.; Prato, M.; Manna, L. Colloidal Synthesis of Quantum Confined Single Crystal $\mathrm{CsPbBr}_{3}$ Nanosheets with Lateral Size Control up to the Micrometer Range. J. Am. Chem. Soc. 2016, 138, 7240. 
(20) Dohner, E. R.; Hoke, E. T.; Karunadasa, H. I. Self-Assembly of Broadband White-Light Emitters. J. Am. Chem. Soc. 2014, 136, 17181721.

(21) Smith, M. D.; Jaffe, A.; Dohner, E. R.; Lindenberg, A. M.; Karunadasa, H. I. Structural Origins of Broadband Emission from Layered Pb-Br Hybrid Perovskites. Chem. Sci. 2017, 8, 4497-4504.

(22) Bohun, A.; Dolejší, J.; Barta, Č. The Absorption and Luminescence of $\left(\mathrm{PbCl}_{6}\right)^{4-}$ and $\left(\mathrm{PbBr}_{6}\right)^{4-}$ Complexes. Czech. J. Phys. 1970, 20, 803-807.

(23) Akkerman, Q. A.; Park, S.; Radicchi, E.; Nunzi, F.; Mosconi, E.; De Angelis, F.; Brescia, R.; Rastogi, P.; Prato, M.; Manna, L. Nearly Monodisperse Insulator $\mathrm{Cs}_{4} \mathrm{PbX}_{6}(\mathrm{X}=\mathrm{Cl}, \mathrm{Br}, \mathrm{I})$ Nanocrystals, their Mixed Halide Compositions, and their Transformation into $\mathrm{CsPb}_{3}$ Nanocrystals. Nano Lett. 2017, 17, 1924-1930.

(24) Liu, Z.; Bekenstein, Y.; Ye, X.; Nguyen, S. C.; Swabeck, J.; Zhang, D.; Lee, S. T.; Yang, P.; Ma, W.; Alivisatos, A. P. Ligand Mediated Transformation of Cesium Lead Bromide Perovskite Nanocrystals to Lead Depleted $\mathrm{Cs}_{4} \mathrm{PbBr}_{6}$ Nanocrystals. J. Am. Chem. Soc. 2017, 139, 5309-5312.

(25) Palazon, F.; Almeida, G.; Akkerman, Q. A.; De Trizio, L.; Dang, Z.; Prato, M.; Manna, L. Changing the Dimensionality of Cesium Lead Bromide Nanocrystals by Reversible Postsynthesis Transformations with Amines. Chem. Mater. 2017, 29, 4167-4171.

(26) Davis, M. M. Acid-Base Behavior in Aprotic Organic Solvents; U.S. Department of Commerce, National Bureau of Standards, 1968.

(27) Ravi, V. K.; Santra, P. K.; Joshi, N.; Chugh, J.; Singh, S. K.; Rensmo, H.; Ghosh, P.; Nag, A. Origin of Substitution Mechanism for the Binding of Organic Ligands on the Surface of $\mathrm{CsPbBr}_{3}$ Perovskite Nanocubes. J. Phys. Chem. Lett. 2017, 8, 4988-4994.

(28) Weidman, M. C.; Goodman, A. J.; Tisdale, W. A. Colloidal Halide Perovskite Nanoplatelets: An Exciting New Class of Semiconductor Nanomaterials. Chem. Mater. 2017, 29, 5019-5030.

(29) Maryott, A. A. Conductimetric Titrations of Acids and Bases In Benzene and Dioxane. J. Res. Natl. Bur. Stand. (1934) 1947, 38, 527536.

(30) Witschonke, C. R; Kraus, C. A. Properties of Electrolytic Solutions. XXIX. The Conductance of Some Electrolytes in Nitrobenzene at 25C. J. Am. Chem. Soc. 1947, 69, 2472-2841.

(31) Coetzee, J. F.; Padmanabhan, G. R. Properties of Bases in Acetonitrile as Solvent. IV. Proton Acceptor Power and Homoconjugation of Mono- and Diamines. J. Am. Chem. Soc. 1965, 87, 5005-5010.

(32) Moreels, I.; Fritzinger, B.; Martins, J. C.; Hens, Z. Surface Chemistry of Colloidal PbSe Nanocrystals. J. Am. Chem. Soc. 2008, 130, 15081-15086.

(33) Moreels, I.; Justo, Y.; De Geyter, B.; Haustraete, K.; Martins, J. C.; Hens, Z. Quantum Dots: A Surface Chemistry Study. ACS Nano 2011, 5, 2004-2012.

(34) De Roo, J.; Ibáñez, M.; Geiregat, P.; Nedelcu, G.; Walravens, W.; Maes, J.; Martins, J. C.; Van Driessche, I.; Kovalenko, M. V.; Hens, Z. Highly Dynamic Ligand Binding and Light Absorption Coefficient of Cesium Lead Bromide Perovskite Nanocrystals. ACS Nano 2016, 10, 2071-2081. 